\title{
Multisoliton regime of pulse generation by lasers passively mode locked with a slow saturable absorber
}

\author{
J. M. Soto-Crespo \\ Instituto de Óptica, Consejo Superior de Investigaciones Cientificas, Serrano 121, 28006 Madrid, Spain
}

\begin{abstract}
N. N. Akhmediev
Australian Photonics Co-operative Research Centre, Optical Science Centre, Research School of Physics Science and Engineering, The Australian National University, Canberra ACT 0200, Australia
\end{abstract}

Received November 30, 1998

\begin{abstract}
We perform a study of soliton generation by a passively mode-locked laser using a slow saturable absorber. We show theoretically that multisoliton regimes of generation are possible. The values of the parameters are found where one-, two-, or three-soliton solutions exist. (c) 1999 Optical Society of America [S0740-3224(99)00504-4]

OCIS codes: $060.4370,060.5530,060.7140,140.7090$.
\end{abstract}

The passively mode-locked laser ${ }^{1-5}$ has been a muchstudied device in recent years. Ultrashort-pulse generation in laser systems makes use of a variety of schemes, including figure-of-eight fiber laser design, ${ }^{1}$ fast saturable absorbers, ${ }^{4}$ additive-pulse mode locking, ${ }^{5}$ and nonlinear polarization rotation. ${ }^{6,7}$ The use of a semiconductor saturable absorber mirror ${ }^{8}$ with a relatively slow response time and an even slower recovery time has been suggested for a passively mode-locked soliton laser. ${ }^{9}$ Experimental verification of this possibility has been reported in Ref. 10.

Pulse-train generation in passively mode-locked lasers has been observed both in regular ${ }^{11}$ and chaotic ${ }^{12}$ regimes. Pulsing behavior with two or three pulses inside the cavity has also been observed. ${ }^{13}$ Usually multiplepulse generation is suppressed so that there is more energy in a single pulse. ${ }^{14}$ However, multipulse laser operation could be useful in a number of applications. In this work we make a theoretical study of the multipulse regime of a solid-state laser with a slow saturable absorber.

It is common practice ${ }^{3}$ to model the laser as a distributed system if the pulse shape changes only slightly during each round trip. The pulse evolution is then governed by a modified complex Ginzburg-Landau equation with nonlinear nonconservative terms ${ }^{9,15}$ :

$$
i \psi_{z}+\frac{D}{2} \psi_{t t}+|\psi|^{2} \psi=i\left[g(\boldsymbol{Q})-\delta_{s}\left(|\psi|^{2}\right)\right] \psi+i \beta \psi_{t t},
$$

where $z$ is the cavity round-trip number (normalized such that $z=1$ corresponds to one round trip), $t$ is the retarded time, $\psi$ is the normalized envelope of the optical field, $D$ is the group delay dispersion coefficient, $\beta$ stands for spectral filtering $(\beta>0), g(Q)$ is the cavity gain $\left(Q=\int_{-\infty}^{\infty}|\psi|^{2} \mathrm{~d} t\right)$, and $\delta_{s}\left(|\psi|^{2}\right)$ represents the losses in the cavity and in the slow saturable absorber.

The gain term $g(Q)$ in Eq. (1) describes a typical solidstate laser-gain medium with a recovery time much longer than the round-trip time of the cavity. For our purposes the recovery time can be considered infinite. Therefore, $g(Q)$, which accounts for gain depletion, depends on the total pulse energy in the following way:

$$
g(Q)=\frac{g_{0}}{1+\frac{Q}{E_{L}}} .
$$

Here $E_{L}$ is the saturation energy and $g_{0}$ is the smallsignal gain. The value of $g(Q)$ decreases as the energy increases; thus only a limited number of pulses can exist inside the cavity.

The loss modulation in the saturable absorber can be described by the following rate equation ${ }^{9}$ :

$$
\frac{\partial \delta_{s}}{\partial t}=-\frac{\delta_{s}-\delta_{0}}{T_{1}}-\frac{|\psi|^{2}}{E_{A}} \delta_{s},
$$

where $T_{1}$ is the recovery time of the saturable absorber, $\delta_{0}$ is the loss introduced by the absorber in the absence of pulses, and $E_{A}$ is the saturation energy of the absorber.

We sought for solutions of the propagation equation and their range of existence, following the method described in Ref. 16. In all simulations we used periodic boundary conditions. Single-pulse solutions of Eq. (1) exist for a certain range of the parameters. Following Refs. 9 and 10 we call them solitonlike pulses or simply solitons. For the pulse to be stable, the gain $g$ must depend on the energy $Q$ as in Eq. (2). The $Q$ dependence of $g$ serves as a feedback mechanism that stabilizes the pulse for a given value of $E_{L}$. When the pump power is high 


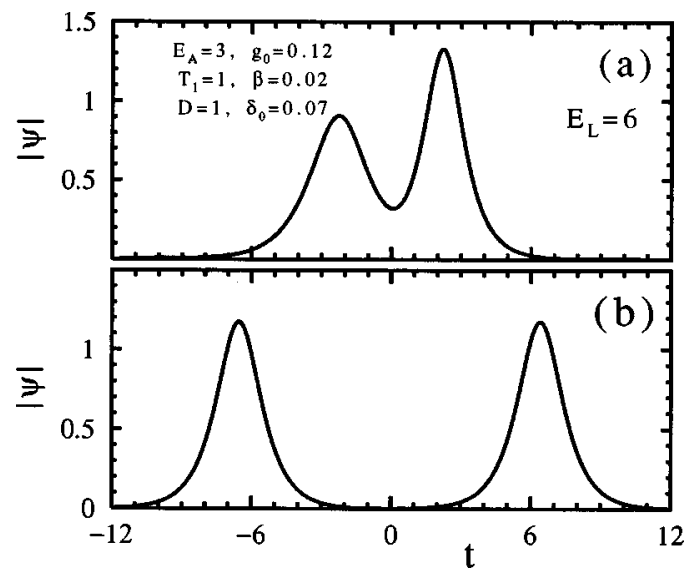

Fig. 1. Stable soliton pairs of (a) type A and (b) type B. The parameters used in the simulation are shown in the figure.

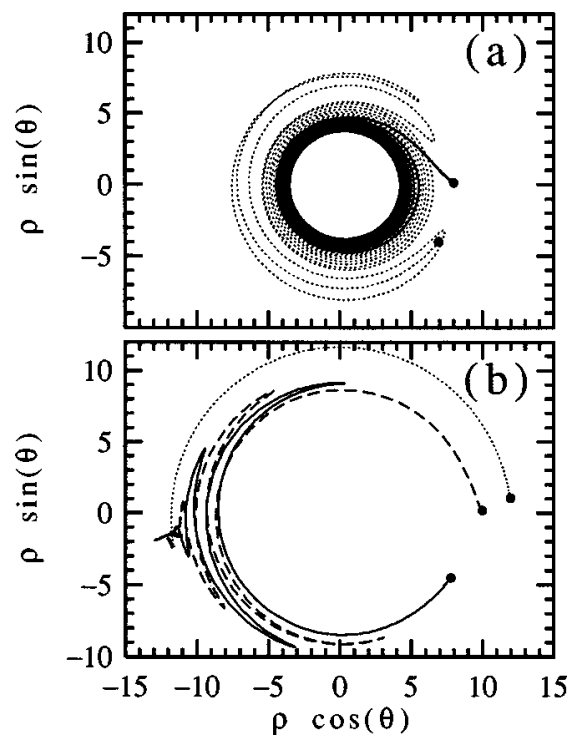

Fig. 2. Trajectories showing the evolution of (a) A-type and (b) B-type two-soliton solutions on the interaction plane; $\rho$ is the distance between the solitons, and $\theta$ is the phase difference. The solid circle shows the starting point. The values of parameters for which we made these calculations are $\delta_{0}=0.07, T_{1}=1, \beta$ $=0.02, g_{0}=0.12, E_{L}=6$, and $E_{A}=3$.

enough, two or more pulses may be generated sequentially. Because the total gain/loss is influenced by the pulses, two coexisting pulses inside the cavity influence each other, and the distance between them can be fixed.

The maximum number of pulses per round trip is determined by the gain parameters $E_{L}$ and $g_{0}$ for otherwise fixed parameters. In the regime where two pulses are possible, they can propagate independently if they are far apart from each other. On the other hand, if the pulses get close to each other, they can form a bound state. The distance and the phase difference between the pulses are determined by complicated dynamics inside the cavity, especially, by the balance between gain and loss. ${ }^{17}$ The new feature of bound states here is that, owing to loss relaxation, the pulse shapes (and their amplitudes) also depend on the relative distance between them. This, in turn, influences the overall balance between gain and loss.
In this paper we study the bound states numerically. Our first observation is that there are two types of bound states for the same set of parameters. One is asymmetric, with unequal pulse amplitudes [see Fig. 1(a)], and the other is symmetric, with equal pulse amplitudes [see Fig. 1(b)]. We denote the former pair as A-type and the latter as B-type.

Solitons in A-type pairs are bound owing to the balance of gain and loss at a certain distance $\rho_{A}$ between the pulses. The pulse amplitudes also vary with the relative distance because the gain/loss difference for the second pulse is different from that for the first. Then, the overall balance is determined by the distance. The phases of the two solitons are independent. Owing to the unequal amplitudes, the rates at which their phases change are different, and then the phase difference between the solitons increases monotonically. Trajectories on the interaction plane ${ }^{17}$ corresponding to these pairs are almost circles around a certain point that is close to the origin but does not coincide with it (see Fig. 2). The shift of the center of the orbit from the origin indicates that the overall balance also depends on the relative phase difference between the two pulses in the bound state: pulses tend to approach when they are in phase and separate when they are out of phase. This periodic change of attraction to repulsion and vice versa results in zero average force between the pulses at certain pulse separation. There is a limit cycle to which all nearby trajectories converge. As a result, the bound state $\mathrm{A}$ is stable.

The two solitons in a B-type pair are located at a distance much larger than the recovery time $T_{1}$. Hence the interaction is small and weakly influences the amplitudes of the two pulses. Nevertheless, the two solitons interact through their tails (the field amplitude between the pulses is $\approx 10^{-2}$ ). Although the interaction is small, its presence is indicated by the fact that the phase difference between the pulses in this case is fixed and close to $\pi$. Owing to the asymmetry of the mutual interaction, the phase difference is slightly less than $\pi$. In this case the equilibrium separation between the pulses is due to the balance between the repulsion of solitons with opposite phases and their attraction through the gain/loss mechanism. The fixed point in this case appears to be the stable focus. Trajectories that start from distances around the equilibrium converge to this singular point (see Fig. 2). The convergence shows that the pair B is also stable.

The bound states that we are discussing are sensitive to the variation of parameters in Eq. (1) and to the initial conditions. Note first that the form of the nonlinearity does not influence their existence much whenever it is close to the Kerr-type. A small higher-order quintic term does not destroy the bound states. Other parameters may have decisive influence. We numerically investigated the range of parameters where asymmetric and symmetric two-soliton solutions exist. In particular, Fig. 3 shows the area in the $\left(E_{A}, E_{L}\right)$ plane where we found these two types of solutions. The dark region is the one where asymmetric bound states of type A appear. Type-B solutions appear in most of this dark area and additionally in the gray area. This zone is bordered by a solid curve. Above the upper boundary of the dark region 
in Fig. 3, only three-soliton bound states appear, and below it only single-soliton solutions exist. There are also three-soliton solutions below the upper boundary and single-soliton solutions above the lower boundary. Furthermore, the behavior of symmetric states depends on the value of $E_{A}$. Specifically, if we keep the saturation parameter $E_{A}$ below $\sim 1.5$, keeping all other parameters the same as before, the motion becomes periodic in $z$ [see Fig. 4(a)]. The period of this motion is $\sim 1300$ round trips for $E_{A}=0.5$ [see Fig. 4(b)]. The phase difference evolves periodically and can rotate in a clockwise as well as in an anticlockwise direction, with two turning points [see Fig.

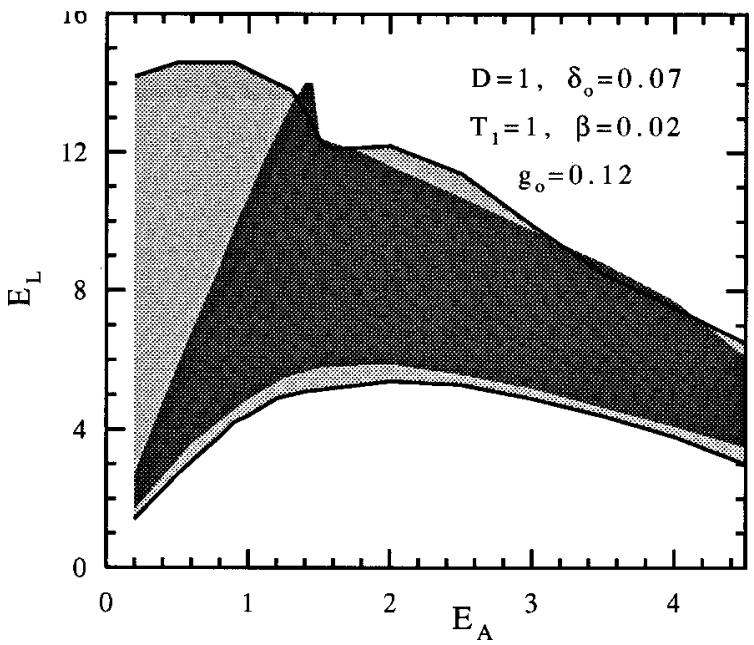

Fig. 3. Regions of existence for two-soliton bound states.
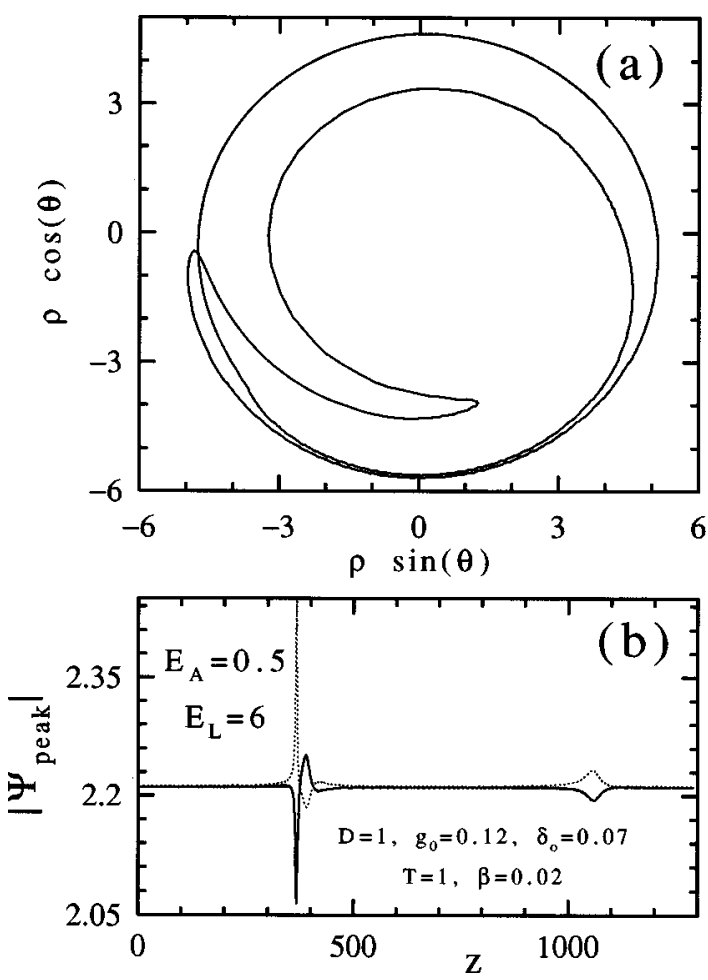

Fig. 4. (a) Trajectories showing the evolution of periodic B-type two-soliton solutions on the interaction plane. (b) Evolution of the amplitudes of the two pulses.

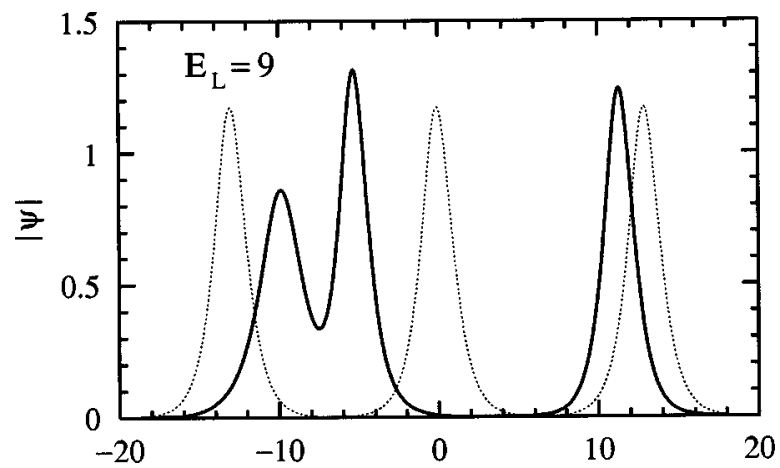

Fig. 5. Stable soliton triplets of two different types. The values of the parameters are the same as in Fig. 2, except $E_{L}=9$.

4(a)]. Clearly, the dynamics depends on the phase difference between the two pulses. The amplitudes of the two pulses are almost equal during the whole period [see Fig. 4 (b)] except at the points $z \approx 400$ and $z \approx 1050$. For these bound states the distance between the pulses also changes periodically.

If the saturation energy $E_{L}$ and/or $E_{A}$ are high enough, and the point is located in the area in Fig. 3 above the two dark areas, then three solitons can exist in a bound state. These multisoliton complexes can be ordered in two types of triplets. Either two pulses are combined into a pair of type A and the third one is at a distance $\rho_{B}$ from them, or the two separations are both equal to $\rho_{B}$ (Fig. 5). Four and more soliton solutions are also possible.

The multipulse generation described above is in qualitative agreement with experimental observations of $\mathrm{B}$. Luther-Davies and M. Lederer made in the Laser Physics Center of the Australian National University. Multisoliton generation is a complicated phenomenon, and further experimental and theoretical investigations are needed.

The authors thank B. Luther-Davies and M. Lederer for initiating this work and for many useful discussions and A. Ankiewicz for help with the manuscript. The work of J. M. Soto-Crespo was supported by the Comunidad de Madrid under contract 06T/039/96 and by the Dirección General de Enseñanza Superior under contract PB96-0819.

\section{REFERENCES}

1. I. N. Duling, Opt. Lett. 16, 539 (1991).

2. S. Gray, A. B. Grudinin, W. H. Loh, and D. N. Payne, Opt. Lett. 20, 189 (1995).

3. C.-J. Chen, P. K. A. Wai, and C. R. Menyuk, Opt. Lett. 19, 198 (1994)

4. H. A. Haus, J. G. Fujimoto, and E. P. Ippen, J. Opt. Soc. Am. B 8, 2068 (1991).

5. H. A. Haus, E. P. Ippen, and K. Tamura, IEEE J. Quantum Electron. 30, 200 (1994).

6. V. J. Matsas, D. J. Richardson, T. P. Newson, and D. N. Payne, Opt. Lett. 18, 358 (1993).

7. M. Hofer, M. E. Fermann, F. Haberl, M. H. Ober, and A. J. Schmidt, Opt. Lett. 16, 502 (1991).

8. M. J. Lederer, B. Luther-Davies, H. H. Tan, and C. Jagadish, Appl. Phys. Lett. 70, 3428 (1997).

9. F. X. Kärtner and U. Keller, Opt. Lett. 20, 16 (1995).

10. I. D. Jung, F. X. Kärtner, L. R. Brovelli, M. Kamp, and U. Keller, Opt. Lett. 20, 1892 (1995). 
11. V. J. Matsas, T. P. Newson, and M. N. Zervas, Opt. Commun. 92, 61 (1992).

12. A. Schwache and F. Mitschke, Phys. Rev. E 55, 7720 (1997).

13. J. A. der Au, D. Kopf, F. Morier-Genoud, M. Moser, and U. Keller, Opt. Lett. 22, 307 (1997).

14. B. C. Barnett, L. Rahman, M. N. Islam, Y. C. Chen, P.
Bhattacharya, W. Riha, K. V. Reddy, and A. T. Howe, Opt. Lett. 20, 471 (1995).

15. H. A. Haus, J. Appl. Phys. 46, 3049 (1975).

16. J. M. Soto-Crespo, N. N. Akhmediev, and V. V. Afanasjev, J. Opt. Soc. Am. B 13, 1439 (1996).

17. N. Akhmediev, A. Ankiewicz, and J. M. Soto-Crespo, Phys. Rev. Lett. 79, 4047 (1997). 